ARTICULO ORIGINAL

\title{
Perfil hematológico del burro criollo (Equus asinus) colombiano
}

\author{
Hematological profile of Colombian creole donkey (Equus asinus)
}

\author{
Herrera B Yonairo ${ }^{1 *}$ MVZ; Rugeles P Clara² M.Sc; Vergara G Oscar² Dr.Sc.
}

${ }^{1}$ Universidad de Córdoba. Programa de Medicina Veterinaria y Zootecnia. Departamento de Ciencias Pecuarias. Grupo de Investigación en Producción Animal Tropical GIPAT. Montería. Colombia.

${ }^{2}$ Universidad de Córdoba. Programa de Maestría en Ciencias Veterinarias del Trópico. Facultad de Medicina Veterinaria y Zootecnia. Grupo de Investigación en Producción Animal Tropical GIPAT. Montería. Colombia.

Keywords:

Hemogram; reference parameters; white blood cells; erythrogram.

Palabras clave:

Hemograma;

parámetros de referencia; glóbulos blancos; eritrograma.

\section{Abstract}

The diagnosis of diseases in animals depends to a large extent on clinical examinations and laboratory results, in which biological variations are reflected. The hematological values are very important for the evaluation of the normal physiological state of an animal. However, very few works have been carried out in this respect on creole asses. The objective of this investigation was to determine the mean values of a hemogram for creoles donkeys of the Colombian lowland. A cross - sectional descriptive study was performed with a non - probabilistic sample for convenience. Samples of 85 male donkeys between 4 and 15 years old were clinically healthy and fed with Brachiaria humidicula pastures. These parameters were obtained by means of a hematological analyzer (ABX Micros ESV 60). The values obtained were: leukocytes $7.6 \pm 1.6 \times 10^{3} / \mathrm{mm}^{3}$, lymphocytes $39.69 \pm 10.87 \%$, monocytes $5.89 \pm 1.63 \%$, granulocytes $54.41 \pm 10.75 \%$, eosinophils $7.08 \pm 2.77 \%$, erythrocytes $4.48 \pm 0.85 \times 10^{6} / \mathrm{mm}^{3}$, hemoglobin $8.62 \pm 1.3 \mathrm{~g} / \mathrm{dL}$, hematocrit $30.24 \pm 5.1 \%$, mean corpuscular volume $67.71 \pm 5.91 \mu \mathrm{m}^{3}$, mean corpuscular hemoglobin $19.45 \pm 1.94 \mathrm{pg}$, mean corpuscular hemoglobin concentration $28.48 \pm 1.36 \mathrm{~g} /$ $\mathrm{dL}$ and platelets $354.20 \pm 136.24 \times 10^{3} / \mathrm{mm}^{3}$. The results confirm that there are differences between the values in most hematological parameters of creole donkeys compared to those of other breeds and horses. These results may be useful in the veterinary clinical routine for the diagnosis, monitoring and control of diseases.

\section{Resumen}

El diagnóstico de las enfermedades en los animales, depende en gran medida de los exámenes clínicos y resultados de laboratorio, en los cuales se reflejan variaciones biológicas. Los valores hematológicos son muy importantes para la evaluación del estado fisiológico normal de un animal. Sin embargo, son muy pocos los trabajos que se han llevado a cabo en este aspecto en asnos criollos. El objetivo de esta investigación fue determinar los valores medios de un hemograma para burros criollos del tópico bajo colombiano. Se realizó un estudio descriptivo de corte transversal con muestro no probabilístico por conveniencia. Se tomaron muestras de 85 burros machos entre 4 y 15 años clínicamente sanos y alimentados con pasturas de Brachiaria humidicula. Estos parámetros se obtuvieron por medio de un analizador hematológico (ABX Micros ESV $60)$. Los valores obtenidos fueron: leucocitos $7,6 \pm 1,6 \times 10^{3} / \mathrm{mm}^{3}$, linfocitos $39,69 \pm 10,87 \%$, monocitos $5,89 \pm 1,63 \%$, granulocitos $54,41 \pm 10,75 \%$, eosinófilos $7,08 \pm 2,77 \%$, eritrocitos $4,48 \pm 0,85 \times 10^{6} / \mathrm{mm}^{3}$, hemoglobina $8,62 \pm 1,3 \mathrm{~g} / \mathrm{dL}$, hematocrito $30,24 \pm 5,1 \%$, volumen corpuscular medio $67,71 \pm 5,91{\mu \mathrm{m}^{3}}^{3}$, hemoglobina corpuscular media $19.45 \pm 1.94$ pg, concentración de hemoglobina corpuscular media $28,48 \pm 1,36 \mathrm{~g} / \mathrm{dL}$ y plaquetas $354,20 \pm 136,24 \times 10^{3} / \mathrm{mm}^{3}$. Los resultados confirman que existen diferencias entre los valores en la mayoría de los parámetros hematológicos de burros criollos comparados con los de otras razas y equinos. Estos resultados pueden ser útiles en la rutina clínica veterinaria para el diagnóstico, seguimiento y control de enfermedades.
Recibido: 23-02-2017;

Aceptado: 20-06-2017.

Correspondencia autor:

yonairo@yahoo.es 


\section{Introducción}

El hemograma también conocido como cuadro hemático, biometría hemática o recuento de células sanguíneas es una de las pruebas que más se solicita al laboratorio clínico, y sin duda alguna, la prueba de laboratorio que más aporta al clínico en la evaluación de un paciente. El hemograma se define como el análisis cuantitativo y cualitativo de los componentes celulares de la sangre periférica (CAMPUZANO-MAYA, 2007).

La determinación de los principales parámetros hematológicos y bioquímicos de los animales ayuda a los veterinarios a confirmar los diagnósticos clínicos, estimar la gravedad de los casos, seleccionar el tratamiento apropiado y evaluar los resultados (ROUBIES et al., 2006). Para interpretar correctamente los datos, los análisis obtenidos en el laboratorio deben ser comparados con los valores correspondientes a los valores de referencia de los animales clínicamente sanos, que sirven como una guía para el médico en la evaluación de dichos resultados (YOKUS et al., 2006).

Debido a su evolución durante miles de años, los burros se adaptaron a climas áridos, adquiriendo la capacidad de permanecer en condiciones ambientales cálidas y secas con más facilidad que los caballos y crearon ciertos mecanismos de adaptación fisiológica distinta a otros animales de su misma especie (MATTHEWS, 2010). Por ende, utilizar la información existente en los equinos y extrapolarla para el manejo clínico en burros, puede no ser una comparación equitativa (de ALUJA et al., 2001). Aunque pertenecientes a la misma familia (équidos) y el mismo género (Equus), caballos y burros presentan características muy diferentes en su eritrogramas (PERDIGÃO et al., 1974). Con base en lo anterior, se planteó como objetivo principal de establecer parámetros hematológicos de referencia para burros criollos colombianos.

\section{Materiales y métodos}

Área de estudio. El estudio se llevó a cabo en predios ubicados en cuatro municipios del departamento de Córdoba, Colombia, ubicado entre las coordenadas $7^{\circ} 23^{\prime}$ y $9^{\circ} 26^{\prime}$ de latitud norte y los $74^{\circ} 52^{\prime}$ y $76^{\circ} 32^{\prime}$ de longitud oeste, a una altura de $30 \mathrm{msnm}$, con temperatura promedio anual de $28^{\circ} \mathrm{C}$, humedad relativa del $84 \%$, precipitación media anual de $1200 \mathrm{~mm}$ y pertenece a la formación climática de bosque tropical lluvioso (CASSAB et al., 2010).

Animales de estudio. Las unidades de muestreo para este estudio fueron 85 burros machos criollos adultos de trabajo de entre 4 y 15 años de edad, clínicamente sanos, criados bajo un sistema de producción extensivo y previamente desparasitados con doramectina (Dectomax, Pfizer, EUA) a dosis única de 0,2 $\mathrm{mg} / \mathrm{kg}$ vía intramuscular. Los animales no recibieron suplementación nutricional, pastaron en praderas donde predominaba Brachiaria humidicola. La edad fue determinada por cronología dentaria siguiendo los parámetros descritos por McCARTHY (1986). Por otra parte, todos los burros en el estudio estaban con condición corporal entre 2 y 2,5 según la escala descrita por SVENDSEN (1997). Los burros entraron en fase de seguimiento durante dos semanas antes de la obtención de la muestra para la determinación de los parámetros hematológicos.

Obtención de la muestra. La toma de muestras de sangre de los animales se realizó por medio de sistema de recogida al vacío (BD Vacutainer $囚$ ) en tubos con anticoagulante (EDTA) con capacidad para $4 \mathrm{ml}$. La colecta se ejecutó, después de la antisepsia apropiada de la región a través de la punción de la vena yugular externa (MEYER et al., 1995). Las muestras se rotularon después de la toma y fueron embaladas en cajas de poliestireno expandido manteniendo una temperatura de $4^{\circ} \mathrm{C}$ para el almacenamiento temporal durante el transporte al Laboratorio de Andrología de la Facultad de Medicina Veterinaria y Zootecnia de la Universidad de Córdoba para su posterior análisis.

Procesamiento de muestra. Las muestras fueron leídas con en un Analizador Hematológico Veterinario (ABX Micros ESV 60, Horibia, EEUU). Las variables determinadas fueron:

Leucograma: leucocitos (x $\left.10^{3} / \mu \mathrm{L}\right)$, linfocitos (\%), monocitos $(\%)$, granulocitos $(\%)$, eosinófilos $(\%)$, linfocitos $\left(x 10^{3} / \mathrm{mm}^{3}\right)$, monocitos $\left(\times 10^{3} / \mathrm{mm}^{3}\right)$, granulocitos $\left(\mathrm{x} 10^{3} / \mathrm{mm}^{3}\right)$ y eosinófilos $\left(x 10^{3} / \mathrm{mm}^{3}\right)$.

Eritrograma: eritrocitos $(x 106 / \mu \mathrm{L})$, hemoglobina $(\mathrm{g} /$ $\mathrm{dL}$ ), hematocrito (\%), volumen corpuscular medio (fL), hemoglobina corpuscular media (pg) y concentración de hemoglobina corpuscular media ( $\mathrm{g} / \mathrm{dL})$. Plaquetograma: plaquetas $\left(\times 10^{3} / \mathrm{mm}^{3}\right)$.

Análisis estadístico: Los resultados obtenidos se tabularon en Microsoft Excel® 2010 y analizados mediante estadística descriptiva para cada una de las variables, utilizando para ello el paquete estadístico SAS 9.1.

\section{Resultados}

Los constituyentes hematológicos del burro criollo colombiano (Tablas 1 y 2), presentan diferencias con otros valores reportados en burros por diferentes investigadores (BABEKER y ABDALBAGI, 2014; GIRARDI, 2012, BURDEN et al., 2015; TESFAYE et al., 2014; GIRARDI et al., 2013; ALVES, 2008; GARBA 
et al., 2015), así como también a datos informados en equinos (Álvarez et al., 2016).

Tabla 1. Valores medios, desvío estándar (DE), coeficiente de variación $(\mathrm{CV})$, valor mínimo y máximo del leucograma en burros criollos colombiano.

\begin{tabular}{cccccc}
\hline Variable & Media & D.E. & CV & Mínimo & Máximo \\
\hline Leucocitos $\left(\times 10^{3} / \mathrm{mm}^{3}\right)$ & 7,60 & 1,60 & 21,0 & 3,60 & 12,80 \\
Linfocitos $(\%)$ & 39,69 & 10,87 & 27,39 & 22,20 & 66,30 \\
Monocitos $(\%)$ & 5,89 & 1,63 & 27,65 & 2,40 & 10,60 \\
Granulocitos $(\%)$ & 54,41 & 10,75 & 19,75 & 28,00 & 73,50 \\
Eosinófilos $(\%)$ & 7,08 & 2,77 & 39,13 & 2,00 & 14,30 \\
Linfocitos $\left(\times 10^{3} / \mathrm{mm}^{3}\right)$ & 2,98 & 1,03 & 34,74 & 0,70 & 6,20 \\
Monocitos $\left(\times 10^{3} / \mathrm{mm}^{3}\right)$ & 0,40 & 0,19 & 46,49 & 0,10 & 1,10 \\
Granulocitos $\left(\times 10^{3} / \mathrm{mm}^{3}\right)$ & 4,26 & 1,25 & 29,35 & 1,90 & 6,70 \\
Eosinófilos $\left(\times 10^{3} / \mathrm{mm}^{3}\right)$ & 0,53 & 0,21 & 39,87 & 0,10 & 1,04 \\
\hline
\end{tabular}

Tabla 2. Valores medios, desvío estándar (DE), coeficiente de variación (CV), valor mínimo y máximo del eritrograma y plaquetograma en burros criollos colombiano.

\begin{tabular}{cccccc}
\hline Variable & Media & D.E. & CV & Mínimo & Máximo \\
\hline Eritrocitos $\left(\times 10^{6} / \mathrm{mm}^{3}\right)$ & 4,48 & 0,85 & 18,95 & 3,08 & 7,22 \\
Hemoglobina $(\mathrm{g} / \mathrm{dL})$ & 8,62 & 1,30 & 15,05 & 6,10 & 12,40 \\
Hematocrito $(\%)$ & 30,24 & 5,10 & 16,85 & 20,40 & 44,60 \\
VCM (fL) & 67,71 & 5,91 & 8,73 & 47,00 & 82,0 \\
HCM (pg) & 19,45 & 1,94 & 9,99 & 13,30 & 26,60 \\
CHCM (g/dL) & 28,48 & 1,36 & 4,78 & 17,90 & 30,50 \\
Plaquetas $\left(\mathrm{x} 10^{3} / \mathrm{mm}^{3}\right)$ & 354,20 & 136,24 & 22,04 & 183,0 & 462,20 \\
\hline
\end{tabular}

\section{Discusión}

Para los valores obtenidos en el eritrograma para burros criollos, es importante resaltar que todos los datos reportados previamente arrojan resultados que se encuentran ubicados por encima de los datos determinados en esta investigación.

La hemoglobina se encontró similar a la reportada en equinos por ÁLVAREZ (2016), pero inferior a la de otros reportes en burros. EI VCM fue superior al reportado tanto en equinos como en burros. La HCM se encontró similar a la reportada por los autores en mención, mientras que la $\mathrm{CHCM}$ se halló inferior. Las plaquetas estuvieron dentro del rango de los reportes realizados por GIRARDI (2012), BABEKER y ADALBAGI (2014).

Las diferencias en los resultados del perfil hemático quizás se pudieron presentar debido a la influencia del estado nutricional de los animales, deficiencias nutricionales en las dietas de los animales afectan el proceso de hematopoyesis en general, principalmente la carencia de vitaminas y minerales (REECE, 1997; GIRARDI, 2012). La regulación de la hematopoyesis es compleja y comprende interacciones entre una célula y otra dentro del microambiente de la médula ósea, así como de factores tanto hematopoyéticos como linfopoyéticos; también requiere aporte adecuado de minerales como hierro y cobre, y varias vitaminas, entre ellas ácido fólico (B9), Cobalamina (B12), piridoxina (B6), ácido ascórbico (C) y riboflavina (B2). Las deficiencias de estos minerales y vitaminas por lo general originan anemias características y con menor frecuencia, insuficiencia general de la hematopoyesis. El hierro, la vitamina B12 y el ácido fólico son los nutrientes esenciales que se requieren para la eritropoyesis normal. El hierro es necesario para la producción de hemoglobina, la vitamina B12 y el ácido fólico son indispensables para la síntesis normal de DNA (JUÁREZ, 2016).

Además, es importante mencionar que la altitud también representa un efecto sobre los indicadores de la eritropoyesis y el metabolismo del hierro, a medida que se asciende sobre el nivel del mar la presión barométrica disminuye y por lo tanto la presión parcial de los gases también lo hace, siguiendo la ley de Dalton (TROMPETERO-GONZÁLEZ et al., 2015). Esta disminución de la presión parcial del oxígeno en el aire genera hipoxia hipóbarica (PATITUCCI et al., 2009). Al producirse estos cambios, los tejidos no reciben las cantidades de oxígeno requeridas para los procesos del metabolismo, por lo cual, el organismo genera estrategias de compensación de manera aguda y si la hipoxia es mantenida en el tiempo — meses, años, generaciones - se generan adaptaciones crónicas (BASU et al., 2007). Ante esta circunstancia la disminución en la presión arterial de oxígeno en el riñón es registrada por las células intersticiales, lo que contribuye a la estimulación de la producción de eritropoyetina. Los niveles de esta hormona aumentan y como resultado dos o tres días después del estímulo, se observa un incremento en el recuento de los reticulocitos en sangre; pero en el hematocrito se pueden apreciar aumentos solo después de 7 a 10 días del estímulo (Beall et al., 2002). Ante tales respuestas de adaptación, los animales que se encuentran a alturas considerables podrían mantener unos niveles superiores de la serie roja sanguínea en comparación con los animales de este estudio en el cual la altitud no supera los 30 m.s.n.m.

Las diferencias presentadas entre los valores del hemograma de este estudio y los reportados en la investigación realizada en esta misma zona estarían afectados por el tipo de métodos utilizados para determinar dichos valores, estos últimos fueron realizados de forma manual y este tipo de procedimientos ofrecen una fiabilidad entre el 75 a 85\% (CAMPUZANOMAYA, 2007), por lo que el margen de error sería mucho más alto comparado al arrojado por analizadores automatizados que dan información mucho más exacta. 
En lo referente al leucograma, el recuento total de glóbulos blancos se ubicó en un valor intermedio en comparación a los valores obtenidos en otros estudios. Es así que fue superior a lo reportado por BABEKER y ADALBAGI (2014) $\left(6,25 \times 10^{3} / \mathrm{mm}^{3}\right)$, quienes determinaron el perfil hematológico en burros bajo régimen de suplementación, en la región del Nilo en Sudán. Entre tanto, fue inferior a lo reportado por GIRARDI $(2012)\left(12,62 \times 10^{3} / \mathrm{mm}^{3}\right)$ y BURDEN et al (2015) $\left(10,11 \times 10^{3} / \mathrm{mm}^{3}\right)$ quienes determinaron intervalos de referencia en burros domésticos brasileros y del Reino Unido respectivamente. En ambos estudios los animales fueron suplementados con raciones de ensilaje y mantenidos en condiciones de pastoreo adecuadas, también tuvieron acceso voluntario a un bloque mineral, lo cual estaría originando tal diferencia, marcada por el tipo de alimentación. De igual forma, el valor absoluto de glóbulos blancos fue inferior a los obtenidos en dos estudios realizados en burros criollos en el departamento de Córdoba, donde los valores promedio para machos fueron de 10,78 x 103/ $\mathrm{mm}^{3}$ y 10,28 x 103/ $\mathrm{mm}^{3}$ (CASTAÑO y KLINGER, 1989; BERROCAL y MUÑOZ, 1989). En este caso, estos valores fueron obtenidos mediante procedimientos manuales, los cuales comparados con los métodos actuales presentaban mayor grado de error en los resultados. El recuento total de leucocitos fue semejante al hallado por GARBA et al (2015) en burros en el noroeste de Nigeria, los cuales eran alimentados con concentrado de grano de sorgo molido, salvado y torta de cacahuete secado, además, tenían acceso a sal y agua a voluntad. Los datos para leucocitos también fueron similares a los obtenidos en caballos criollos colombianos, estudio realizado en fincas de tres municipios del departamento de Córdoba (ÁLVAREZ et al., 2016).

El valor porcentual de los linfocitos se encontró similar al reportado en equinos por ÁLVAREZ et al (2016) e inferior al encontrado en burros por GIRARDI (2012), BABEKER y ADALBAGI (2014) y BURDEN et al (2015); así como también al reportado en burros en el departamento de Córdoba por CASTAÑO y KLINGER (1989) de 49,9\%.
Los monocitos se encuentran en un nivel ligeramente inferior al hallado en burros y caballos por BABEKER y ADALBAGI (2014) y ÁLVAREZ et al (2016) de 6,8 y $6,13 \%$, respectivamente. Mientras que se ubicaron en un nivel superior a los determinados en burros por GIRARDI (2012) (2,67\%) y BURDEN et al (2015) (3,0\%). Este resultado se podría explicar por la constante exposición de los animales del estudio a situaciones de permanentes lesiones o patologías de carácter inflamatorio e infeccioso que originan un aumento de estas células (MAIR, 1996). La monocitopenia o disminución en el número de monocitos circulantes no tiene relevancia clínica (GRONDIN y DEWITT, 2010; SELLON, 2004).

Los granulocitos en general estuvieron superiores a todos los datos reportados en burros y equinos, de éstos, los eosinófilos fueron inferiores al valor encontrado por BABEKER y ADALBAGI $(2014)(7,8 \%)$ y superior a otros reportes de 4,98, 3,83 y 4,00\%, respectivamente (ÁLVAREZ et al., 2016; GIRARDI, 2012; BURDEN et al., 2015). Esto último, se atribuye al efecto de la interacción antígeno-anticuerpo por parásitos que producen la sensibilización de los eosinófilos con su respectivo aumento circular en sangre periferia (CYWINSKA et al., 2010), debido a la falta de planes antiparasitarios adecuados a estos animales y por ello tienden a mantener la línea de eosinófilos alta. Esto podría explicar el valor hallado por CASTAÑO y KLINGER (1989) de 12,12\% para eosinófilos que comparado con los demás reportes fue alto ya que los animales de ese estudio no tuvieron ningún manejo antiparasitario antes de la toma de muestras.

\section{Conclusión}

Los valores de los constituyentes hematológicos y bioquímicos de los burros criollos colombianos difieren de en su mayoría a los reportados por otros investigadores, tanto en estudios realizados en burros, como en estudios referenciados en equinos. Los resultados del presente estudio podrán servir como valores de referencia para la interpretación de parámetros hemáticos de uso clínico en el diagnóstico de enfermedades de estos animales.

\section{Referencias}

ÁlVAREZ, J.; RUGELES, P.; BRUNAL, E.; CAMPILLO, J. y HERRERA, Y. En imprenta. Parámetros hematológicos en caballos (Equus caballus) de vaquería.

ALVES, L.M. 2008. Influência da idade e do sexo sobre o perfil bioquímico sérico de jumentos da raça brasileira. Tesis. Uberlândia, Brasil: Universidade Federal de Uberlândia, Facultade de Medicina Veterinaria e Zootecnia. Brasil.

BABEKER, E.A.; ABDALBAGI, Y.M. 2014. Hematological profile of donkey (Equus asinus) breed in white Nile State, Sudan. Scientific Journal Issue 10:218-228. 
BASU, C.K.; BANERJEE, P.K.; SELVAMURTHY, W.; SARYBAEV, A.; MIRRAKHIMOV, M.M. 2007. Acclimatization to high altitude in the Tien Shan: a comparative study of Indians and Kyrgyzis. Wilderness and Environmental Medicine 18 (2):106-110.

BEALL, C.M.; DECKER, M.J.; BRITTENHAM, G.M.; KUSHNER, I.; GEBREMEDHIN, A.; STROHL, K.P. 2002. An Ethiopian pattern of human adaptation to high-altitude hypoxia. Proceedings of the National Academy of Sciences 99 (26):17215-17218.

BERROCAL G.A.; MUÑOZ, P.J. 1989. Determinación del hemograma en asnos en condiciones naturales en el Sinú medio del departamento de Córdoba. Tesis de pregrado. Montería: Universidad de Córdoba, Ciencias pecuarias. Montería, Córdoba.

BURDEN, F.A.; HAZELL-SMITH, E.; MULUGETA, G.; PATRICK, V.; TRAWFORD, R.; BROOKS BROWNLIE, H.W. 2016. Reference intervals for biochemical and haematological parameters in mature domestic donkeys (Equus asinus) in the UK. Equine Veterinary Education 28 (3):134-139.

CAMPUZANO-MAYA G. 2007. Del hemograma manual al hemograma de cuarta generación. Medicina \& Laboratorio 13:511-550.

CASSAB, A.; MORALES, V.; MATTAR, S. 2010. Factores climáticos y casos de Dengue en Montería, Colombia. 2003-2008. Rev. Salud pública 13 (1):115-128.

CASTAÑO A.T.; KLINGER, B.O. 1989. Determinación del hemograma en asnos (Equus asinus) en condiciones naturales en el bajo Sinú del departamento de Córdoba - Colombia. Tesis de pregrado. Montería: Universidad de Córdoba, Ciencias pecuarias. Montería, Córdoba.

CYWINSKA, A.; WYSZYNSKA, Z.; GÓRECKA, R.; SZARSKA, E.; WITKOWSKI, L.; DZIEKAN, P.; SCHOLLENBERGER, A. 2010. The effect of the $162 \mathrm{~km}$ endurance ride on equine peripheral blood neutrophil and lymphocyte functions. Polish journal of veterinary sciences $13(2): 279$.

De ALUJA, A.S.; BOUDA, J.; LÓPEZ, A.C.; CHAVIRA, H.H. 2001. Valores bioquímicos en sangre de burros antes y después del trabajo. Vet. Mex. 32(4): 271-278.

GARBA, U.M.; SACKEY, A.K.B.; IDRIS, L.A.; ESIEVO, K.A.N. 2015. Baseline vital, haematological and serum biochemical parameters of donkeys. Journal of Veterinary Medicine and Animal Health 7( 3):94-98.

GIRARDI, A.M.; MARQUES, L.C.; DE TOLEDO, C.Z.P.; BARBOSA, J.C.; MALDONADO, J.R.W.; JORGE, R.L.N.; DA SILVA NOGUEIRA, C.A. 2014. Biochemical profile of the Pêga donkey (Equus asinus) breed: influence of age and sex. Comparative Clinical Pathology 23 (4):941-947.

GIRARDI, A. 2012. Parámetros hematológicos e bioquímicos séricos de jumentos (Equus asinus) da raça pêga. Tesis de maestría. Jaboticabal, São Paulo. Brasil: Universidade Estadual Paulista "Júlio de Mesquita Filho".

GRONDIN, T.M. 2010. Normal hematology of the horse and donkey. Págs. 821-828. En: Weiss D.J WKE. Schalm's Veterinary Hematology. Wiley Blackwell Inc.

JUÁREZ, L.M. Revisión Farmacológica. UNAM. [Online].; 2016. Disponible en: http://www.facmed.unam.mx/deptos/ familiar/atfm123/revfarma.html. Consultada: 12-09-2016.

MAIR, T.S. 1996. Update on infectious respiratory diseases of the horse. Equine Vet Educ. 8:329-335.

MATTHEWS, N. 2010. Donkeys - not just small horses. In: NORTHAMERICAN VETERINARY CONFERENCE, 24., 2010, Orlando (USA). Proceedings. Gainesville: North American Veterinary Conference: 214-216.

McCARTHY, E. 1986. The principles and practices of feed rationing for donkeys. In McCARTHY, E. The professional Handbook of the Donkeys.: Svendsen E.D. 
MEYER, D.J.; COLES, E.H.; RICH, L.J. 1995. Medicina de laboratório veterinária: interpretação e diagnóstico. Roca. São Paulo, Brasil.

PATITUCCI, M. 2009. Angiogenic/lymphangiogenic factors and adaptation to extreme altitudes during an expedition to Mount Everest. Acta Physiol. 196(2): 259-265.

PERDIGÃO de OLIVEIRA, F.R.; AUGUSTO, C.; GRASSO, P.L.; SOUZA, H.; BAUDET, G.J. 1974. Eritrograma normal de jumentos Equus asinus das raças Puro-sangue Italiana e Brasileira de 1 a 2 anos de idade. Bol. Ind. Anim. 31 (2):325-329.

REECE, W.O. 1997. Physiology of Domestic Animals. Reece, W (ed.). Williams \& Wilkins. New York.

ROUBIES, N.; PANOUSIS, N.; FYTIANOU, A.; KATSOULOS, P.D.; GIADINIS, N.; KARATZIAS, H. 2006. Effects of age and reproductive stage on certain serum biochemical parameters of Chios sheep under Greek rearing conditions. Journal of Veterinary Medicine Series A 53 (6):277-281.

SELLON, D.C. 2004. Disorders of the hematopoietic system. In Reed SM BW. Equine Internal Medicine. Saunders: Sellon DC Eds. 721-768.

SVENDSEN, E.D. 1997. Parasites Abroad. P'gas. 227-238. En: SED (ed). The Professional Hand Book of the Donkey. 3rd ed. Whittet books. London.

TESFAYE, T.; MAMO, G.; ENDEBU, B.; ABAYNEH, T. 2014. Comparative serum biochemical profiles of three types of donkeys in Ethiopia. Comp Clin Pathol 23:205-212.

TROMPETERO-GONZÁLEZA et al. 2015. Efectos de la exposición a la altura sobre los indicadores de la eritropoyesis y el metabolismo del hierro. Rev. Fac. Med 63 (4):717-25.

YOKUS, B.; CAKIR, D.U.; KANAY, Z.; GULTEN, T.; UYSAL, E. 2006. Effects of seasonal and physiological variations on the serum chemistry, vitamins and thyroid hormone concentrations in sheep. Journal of Veterinary Medicine Series A 53 (6):271-276. 